\title{
Empirical Analysis of a Distributed Software Development Project
}

\author{
Przemyslaw Cichocki and Alessandro Maccari \\ Nokia Siemens Networks, \\ Sienna, 39, 00833 Warsaw, Poland \\ \{przemyslaw.cichocki, alessandro.maccari\}@nsn.com
}

\begin{abstract}
In spite of the abundant research that promotes different methods for software development, and the current method war amidst agile and disciplined methods, little research is done to actually figure out whether real projects, carried out in industrial environments, benefit more from either approach. This paper analyses a real project team's opinions and feelings about project management techniques, software development methods and cultural difference in a multi-site project where traveling and communication are made difficult by restrictions and low-quality infrastructure. The different sites also worked in different time zones and with different working week patterns. The project team members almost unanimously indicated that the presence of a local team leader with authority and flexibility to cover a role that is not exactly as assigned in the beginning, is key factor for the success of this sort of projects. While there was no consensus on whether the project was agile or disciplined, evidence seems to hint towards a more disciplined approach, probably as a compensation for the higher degree of uncertainty that derives from the distributed setup. While the findings of the case study cannot be extended to other organizations without caution, we do infer a number of conclusions on cultural differences, project management tools and techniques.
\end{abstract}

Keywords: Agile, Distributed development, Project management techniques evaluation, Human factors.

\section{Introduction}

Current research on software development methods regularly produces a large amount of material, such as proposals for new software project management methodologies, variations to existing software development methods, enhancements to tools, suggestions for improvement of good practices, and so on.

However, surprisingly little effort is spent on trying to apply research findings to practical case studies and document the feedback thereby obtained for the use of the community. This is probably for either of two reasons: the industrial world does not apply the latest findings of research, or otherwise does not have the time or occasion to report on the findings, which are mainly used (if at all) inside the company where the researchers work.

Agile software development methodologies (like Scrum or Extreme Programming) promised to improve the way we develop software in industry. By enhancing communication 
and putting individuals, rather than processes or tools, in the headlight, agile methods have brought us closer to the fulcrum of software development: the human being, with his talents, defects, inconsistencies and creativity.

However, agile methodologies work best in small teams that are co-located, or at least they can communicate easily and without boundaries. Whether they can be applied to distributed project teams is still a subject of research. It is also not clear whether agile methodologies can be applied as effectively in teams where cultural differences are vast, and where the working day and week do not overlap completely.

From common sense, it is not without a reason that a number of methodologists advocate usage of more structured and disciplined methodologies [2] in situations such as the one we take into consideration in the paper.

We present the results of a research that is focused on practical application of software project management and software development techniques in a distributed team setup. The team was distributed across three sites in two different cities, each located in a different country. Traveling between the countries was made difficult by strict visa regulations, and the quality of international telephone lines was generally low. The customer and the project manager were located in one of the cities, while the main development centre was located in the other city, alongside with some $70 \%$ of the members of the development team.

In order to analyze the impact of the team distribution on the performance of the members of the team, as well as their opinion on the effectiveness of certain software development and project management methodologies and techniques, we carried out a series of interviews with all the individuals that worked on the team for more than two months.

We present a number of findings from our research, and make statements on validity threats, as well as on applicability of the findings to similar organizational environments.

We do not claim that our research is complete. In fact, we believe that it poses the basis for a family of experiments, as advocated by [4], aimed to characterize with greater detail the phenomenon under study.

\section{Research Background}

\subsection{Motivations}

The issues that originate the need for this research are the following:

- The lack of rigorous experimental data on the industrial validity of certain project management and software development methodologies and techniques: what techniques do real software developers value most, and why?

- The uncertainty regarding the applicability of any of the known methods and tools to a fully distributed team, which works on tight deadlines and with limited possibilities to interact: will agile methods be more effective than structured ones in this sort of case?

- The absence of definitive findings from existing research on whether an agile approach to project management produces better results (and is better appreciated) 
than a structured, disciplined approach, especially in the case of distributed project team that works in a deadline-driven business environment.

- The insufficient number of studies that aim to understand how the usage of certain project management techniques and methods affects the effectiveness of the software developers (as perceived by them) and of the entire distributed team.

Most of these issues could be restated without limiting the scope to the organization where the research work was carried out, or even to the entire telecom software development domain as a whole.

However, the scope of this study will be limited to the organization where the project team under study operates. We believe that a small study such as this one cannot be generalized without exercising a lot of care, and that the conclusions of this research should in general only be deemed valid within the specific environment under consideration. Additional remarks on the validity of the study are made towards the end of this paper.

\subsection{Research Goal}

This research work aims to answer the following high-level research questions:

What software development methods and tools are deemed to be most effective in a distributed software development team?

What project management techniques and personal qualities of the project manager are most useful in the environment where the project team under study had to operate?

How did cultural differences influence the project team's life, and how can project management methodologies maximize the positive (or minimize the negative) impact of cultural differences?

How agile or disciplined is this sort of project deemed to be, and how is this judged by the project team members?

\subsection{Research Philosophy and Approach}

Orlikowski and Baroudi provided an excellent classification of philosophies and approaches for information technology research [5].

Following their reasoning, we may list the following facts as characterizing our research work.

- Ontologically, we do not make any assumption on the behavior of project team members, nor on the reflections of project management or software development methodologies on the project organization. We assume that such knowledge is unknown, and try to deduce it from appropriate analysis of collected data.

- Socially, we do not assume any predefined regularity to rule the social reality where the project team members work (and thus live). Anyone who has been involved in software development for a telecom industry can confirm that this assumption is true in most cases!

- Epistemologically, we believe that the phenomena of interest (for instance, the relationship between team members, the consequences of adoption of certain project management techniques and methods, and the intrinsic agility of the project) 
can be understood by in-depth enquiries made with the development teams. From such facts, our research work can be classified as interpretive.

The following sections outline the organization of the project team (research environment), the empirical study design, and the method of collection of the information. We also present our initial answer to the research problems stated before.

\subsection{Project Team Environment}

The project team operates in a real, industrial environment within Nokia Siemens Networks, a major provider of telecommunication infrastructure and services, within a single project. The purpose of the project is to implement and deliver a charging and mediation solution for a customer located in the Middle East. The majority of the project tasks consist in implementing custom add-ons or new features on top of an off-the-shelf product platform.

Normally, in the charging and mediation domain it is difficult to reuse software from other, similar projects that have been carried out in the past. This is due to the fact that charging business models (and thus the technical requirements for the software solution) vary substantially from customer to customer, and it is often cheaper and more convenient to implement such features from scratch rather than reusing work done before. We believe that the all-too-famous NIH (Not Invented Here) syndrome has not played a substantial role in these decisions.

The project under study is part of a larger programme that delivers a series of value added service (VAS) solutions to the same customer. The project team environment is typical of a large company that delivers critical software solutions to a customer that operates in emerging markets: largely driven by deadlines that are so tight to seem unrealistic, and in a generally unstable environment where requirements change often, access to common resources is limited due to the lack of infrastructure, and pressure on the project team members is applied by several stakeholders (both within and without the organization that employs them), and not always with the knowledge or approval of the project manager.

From the technical point of view, the purpose of the mediation solution is to collect Call Data Records (CDR) from different network elements (like mobile switches, GPRS nodes, MMS center. etc.), process their content according to the customer requirements and send them in a format that is readable by the customer's billing system. The solution is based on a certain Nokia Siemens Networks product, on top of which our team implemented software that enabled processing CDRs as per the customer requirements.

At the time when the research work was carried out, the project had lasted around 12 months, and around 1300 man working days had been spent on it. It employed 12 people, out of whom 9 were interviewed for this research.

The team consists of one project manager, one or two technical architect (who own the technical solution and had decision power on the technical architecture) and software engineers numbering between three and eleven. The number of software engineers has varied over time according to the need of the project and according to the number of tasks that have had to be carried out concurrently. The project manager can allocate and release technical resources on a relatively short notice. 
Most engineers, who have been assigned tasks that range from implementation to testing and from documentation to deployment at site, belong to two contractor companies that were based in the same city (but in different buildings) in the European Union. One of the technical architects is co-located with the engineers. The project manager is based in the same city in the Middle East as the customer. Depending on need, a number of engineers have been based in the Middle East as well. On average, approximately the project staff was based in Europe at a given point in time, with the remaining half being based in the Middle East.

Travel between the two locations has been encouraged by the project manager, and sufficient budget has been allocated for the project team to travel between the two sites. However, visa restrictions and tight project delivery deadlines have advised against excessive travel.

The quality of the telephone network between the two countries that host the project staff is generally low, which discourages the usage of phone calls as a frequent communication means. In the Middle Eastern location, the quality of internet service is also somewhat low.

The project team included people of four different nationalities. However, the majority of the technical staff (architects, engineers) shared the same nationality and mother tongue. The customer team and other internal stakeholders belonged to a large number of cultures and nationalities. The English language was normally used for communication between the team members and with most project stakeholders.

Customer and Nokia had to build new organizations from scratch. Although Nokia was already present on the market it was only the mobile phone market and the Nokia Networks (part of Nokia dealing with core network - since April 2007 it became part of the Nokia Siemens Networks) was absent in the customer country.

Setting up the companies it is always a big challenge. In this case it was extremely hard because of tight schedules and lack of resources. Both companies had to attract employees not only from customer's country but virtually from all over the world.

Organizational structure was well defined at high level in both cases and borders of responsibility were clearly marked. However when it came to step down into organizational chart it turned out that there were many communications problems. Due to high pressure coming from tight schedules people were overloaded with work and sometimes it was impossible to get the needed information immediately. This lead to delays and in the end in giving up some of the project cycle phases (e.g. performance tests) in order to meet the deadline. This situation applies not only to relation Nokia Siemens Networks - customer but also internally. For example customer's IT department had problems with proper communication with customer's marketing, in Nokia Siemens Networks happened that one team has changed network device settings without notifying other teams that relied upon those configuration.

All of this forced the project team subconsciously to adjust to the major principle of Agile Manifesto - embrace the change [1]. Although that project was following the certain process (classical approach: requirements gathering, solution proposal, implementation, testing, deployment) the team was aware that despite the fact that requirements was signed off the customer can change it at any moment. Obviously project manager was trying to avoid that situation and teach customer that it should 
follow the certain rules (by change request process for example) but sometimes it was really inevitable - in the end it was our customer and project team should make any effort to fulfill its needs.

\section{Survey Characterization}

This research work is carried out by means of a survey that involved the majority of the team members who had technical roles (e.g. software developer, testing engineer, requirement engineer, technical architect). The survey is articulated into nine questions, grouped in three categories:

1. Method and tools, including two questions that cover generic aspects of the applied software development method.

2. Project management techniques, including four questions that cover specific aspects of project management.

3. Cultural differences, including three questions that focused on the impact of cultural differences (mostly, the difference between European and Middle Eastern culture).

When designing the survey, we put particular emphasis on structuring questions so that respondents would be encouraged to give a lot of details, and limited interruptions even when the interviewer felt that the response was drifting out of the original scope.

Questions were structured in an open way, which is typical of interpretive research. We made every effort to avoid guiding the respondent towards a specific answer, or towards a yes/no answer. When the answer was brief (e.g. when someone replied in the lines of "everything worked well") we tried to ask further questions, trying to dig out a more detailed opinion.

Most of the respondents were interviewed face to face, with the exception of two respondents who were interviewed remotely by means of email. Interviews lasted around one hour each, and were conducted by both authors of this paper.

\section{Results}

Below the result of the survey are presented. The interview was conducted with nine team members that were involved into project activities for two months at least.

\subsection{Methods and Tools}

This part of the survey was dealing with techniques and means that team found useful for development and information sharing in the remotely managed project setup.

Most of the team members were contractors so they were originally employed by the external companies (there were two external companies involved in this project) and then indirectly hired by Nokia Siemens Networks. Due to transition period (Nokia Networks was transforming into Nokia Siemens Networks) it was sometimes hard to create the Nokia Siemens Networks accounts (e-mail, intranet access) for those external employees. This was one of the major factors that people felt prevented them 
from being fully productive during the project. People did not have access to documentation and other resources like software updates and patches which ended up in problems with keeping project deadlines.

The most difficult and challenging part of every project phase was the scope definition because of the communication problems mentioned above. During the implementation the main stress was put on the proper configuration of the mediation device and testing. There was not much pure software development - mostly simple $\mathrm{C}++$ and shell script development tasks. For this reason, the first question (asking which software development techniques helped most in a remote setting) was answered in an insufficient manner. Either there was no answer or the question was misunderstood by the survey respondents. However, one of the team members found the Extreme Programming technique (pair programming) as useful during the deployment and functional testing.

The second question in this section was referring to the tools that were found useful in the information and knowledge sharing. Perhaps not surprisingly, the most efficient way of communication was deemed to be face to face meetings. Despite the fact that so-called modern channels of communications (fixed and mobile telephony, internet) were at every team member's disposal, this classical way of exchanging information was found as the most reliable and effective.

Unfortunately, this way of communication could not be used very often from the obvious reasons (distance, difficulty in travel, cost), so people have used email and chat for their daily communication.

In particular, email was recognized to be a more formal way of communication. Typical cases when email was mentioned to be effective are getting approval on documents or requirements from the customer (where an email message constitutes a sort of contract), and broadcasting of information (e.g. meeting minutes) to the entire project team. Some respondents questioned the effectiveness of the usage of email for person-to-person communication.

Chat, instead, was deemed more effective and useful for daily, informal information exchange. A typical use case is when random questions must be asked to a certain expert about certain software functionality.

Version control systems (which the project used for documentation and source codes) were also deemed helpful in information sharing. Unfortunately, most of them required access to our company's intranet account, which, as explained in the first paragraph of this chapter, often meant that access to such resources could not easily be achieved by some of the team members.

Finally, we prepared a Wiki website [3], to be used by the entire project, where the project phases were briefly described and the latest documentation (requirement specifications, solution descriptions, test cases, project management plan) was available for download. Wiki was listed also as one of the tools that contributed to better knowledge sharing within the team, although it was mostly perceived as a placeholder where to find project documentation, and not so much as a place where people can actively (by editing the Wiki pages for example) influence the way the knowledge is shared. 


\subsection{Project Management Techniques}

The second part of the survey tries to understand how the project management techniques and method that have been used in this project were perceived by the team members.

The first question in this section is about the project management techniques that did succeed in the interaction between the team, customer and project manager located in different areas. It turned out that it is extremely important for most team members to have a delegated person that would act as a local team leader in every site where the team operates. That person should represent the project manager locally and should be able to take proper actions once the situation requires them (e.g. tensions with customer during project manager absence) and is accountable for their consequences. Additionally he or she should also fulfill a role of the communication gateway to the project manager but need of this was not as strongly desired as the local leadership role.

Another question asked about the general characteristic of the project, focusing on whether people thought it was disciplined or agile. Rather surprisingly, there was no common ground in that matter between the team members. Some engineers claimed that project was agile and some were claiming that it was very (too) disciplined. There was also no pattern in the answers with distinction to the assigned role in the project. The conclusion that we make out of this is that perhaps the concept of agility (and, correspondingly, that of discipline) is not perceived in the same way by people.

The third question asked the team which of the project management phases (distinguished according to the PMI model) was made harder than usual in this project by the distributed team setup. It was pointed out that especially the scope management phase was more difficult to accomplish. This phase needed traveling as it required meetings with customer in order to define the requirements for the given project phase. Due to restriction mentioned earlier (visa, different time zones, shifted weekends) this task was performed very often under time pressure. Team members felt that efficient scope and requirement management could not be carried out at a distance, especially during the requirement elicitation phase and during the inevitable project phases when the customer points towards a scope creep.

The last question of this section asked which personal qualities of the project team members were most helpful in a remote project setup. Not surprisingly, trust and commitment were listed most often in this case. People found it extremely comfortable to work in an environment where everybody could count on other team members to help in case of issues. Also, the fact that some of the team members (e.g. technical architect) acted as a local project manager for the team was deemed important: this allowed people to have clarity about task assignments, and it was possible to make important decisions quickly. Some people pointed out that having a competent substitute person (when one of the key person for the project was on leave - e.g. project manager) is crucial.

It is not easy to extract unique conclusions from this set of answers. However, there seems to be evidence that the members of this team deem a more disciplined approach (rather than a more agile one) necessary in this kind of setup. The emphasis that was put in scope management, and the clearly expressed need for a local team leader seem to point in the direction of a higher amount of discipline. Similarly, the fact 
that trust and commitment were deemed to be the most useful personal qualities for team members points to the fact that the very nature of agile projects (based on fast prototyping, trial and error and continuous requirement negotiation) does not fit a distributed team setup such as the one we implemented for this project.

\subsection{Cultural Differences}

In the third part of the survey, we investigated the team members' opinion on the cultural differences in the multinational environment that the project had to work in.

The first question concerned the cultural differences that could be spotted as far as the work approach is concerned. Shifted working days were pointed here as the most obvious difference. They are only three working days that overlap in Europe and Middle East (Monday, Tuesday and Wednesday), and therefore activity planning should take that into account. It happened sometimes that people in both countries have to work overtime in order to finish their task in timely manner.

The issue of mother tongue was mentioned by several team members is not exactly falling into cultural difference category but is worth mentioning. It was noticed that for none of the team members English was a mother tongue. On one hand it was found as an advantage as there was no need of strictly applying the grammar rules, proper vocabulary which made communication process easier. On the other hand the knowledge level of English between team members varied and it was pointed that sometimes it could cause communication problems as well.

The second question queried on how cultural differences influenced everyday activities. The surprising result here is that nobody felt the need to point out any cultural factor that would be disturbing or (even more surprising) stimulating. In two or three cases it was mentioned that a multinational team caused people to be more patient and understanding to other team members. People understood that different nationalities can have different approach to work in terms of pace and quality, and had to adjust their expectations correspondingly.

The last question asked people what they thought should be changed in current project setup in order to benefit more from the cultural differences. One of the issues that surfaced here was the need for careful project management planning. This involves taking into account the shifted working days, leaving enough time for crossteam communication and reviews, allowing people to travel between sites when absolutely necessary, and so on.

The conclusion we may infer from this section is that the fact that the team was distributed among two different sites was judged to be more relevant than the fact that the team had people coming from different cultures and countries. There seemed to be no tangible consequence of cultural difference on daily work.

\section{Validity}

\subsection{Internal Validity}

Internal validity is the extent to which the survey results can be extended to similar projects within the organization where the case study was carried out. 
It seems like the very specific setup of the project makes it harder to find the general pattern that could be applied to other cases. Several key factors (such as different time zones, customer organizational inefficiencies, project team divided in three sites, visa restrictions in customer country, etc.) are characteristic to this particular case, and make the results of this survey hard to generalize, even inside our own organizational environment.

However, there are some general conclusions that can be drawn from this setup and that we believe may apply to other similar projects in our organization. These include at least the following.

- Need of a local team leader in every site.

- Necessity of good information sharing tools.

- Flexibility in project task assignment (medley of roles).

When generalizing the other results that we explain above, we believe that we should exercise caution, even internally. Further research is definitely needed before safe statements can be made in this respect.

\subsection{External Validity}

External validity concerns the applicability of the survey results to different organizations, countries, teams and domains.

All the considerations that we have made for internal validity obviously hold when considering external validity. Actually, we believe that even better care should be exercised when extending the validity of the research outside the boundaries of our organization or domain.

The environment where this project operates is fairly unique, as it involves a very aggressive customer (mostly, in terms of deadlines), an organization (Nokia Siemens Networks) that is relatively new to the country where the customer resides, a blend of experience from different fields inside the project team, and a relatively young average age.

For these reasons, we feel that further research is required before any claims are made about external validity. A series of similar case studies should be repeated across different companies, countries and domains, and results compared before any generic claim is made.

\section{Conclusions}

The analysis of the survey that was conducted among the team members gives indirect clues about what can be deemed helpful in this sort of project setup. We now try to answer the four research questions that were at the base of carrying out this case study.

1. What software development methods and tools are deemed to be most effective in a distributed software development team? Our research cannot give a definitive answer to this question, as there has been little agreement among responders. The issue 
requires further research, though we can assert to some degree of certainty that the usage of each tool (chat, email, phone conference, web sites) has to be disciplined in order to avoid generating conflicting messages or annoyance.

2. What project management techniques and personal qualities of the project manager are most useful in the environment where the project team under study had to operate? From the results of our research, we can state that in such environment having a team leader in every site, and being able to trust other team members are by far the most valued aspects. A blend of usage of different tools (email, teleconference, chat, etc.) for different purposes can also solve most of the information sharing issues. The team's emphasis also fell on scope management, which may be an indication that in distributed and dynamic environments where many variables are subject to sudden change, and the possibility to control people is low, effective management of scope is regarded to be the balancing power that makes projects successful.

3. How did cultural differences influence the project team's life, and how can project management methodologies maximize the positive (or minimize the negative) impact of cultural differences? From the results of this research, it appears that cultural differences do not play a major role in this sort of environment, save perhaps for the mother tongue. Actually, evidence points towards the conclusion that cultural differences can even be stimulating, and increasing the productivity and creativity of the people that are involved in the project. This definitely advocates the need for further research in this area, involving experts in sociology and psychology.

4. How agile or disciplined is this sort of project, and how is it perceived by the project team members? From the answer to one specific question we infer that there is no agreement on whether the project was agile or disciplined, which could point out to different interpretation of the concepts of agility and discipline. However, there seems to be evidence that points towards a more disciplined approach, as this guarantees better scope management, more effective assignment of management roles to different sites and emphasizes trust and commitment.

What made this project successful? It is hard to draw an itemized list of success factors in this case. Undoubtedly, it was a mixture of project management techniques, tools and the unique personalities of the people involved in project activities.

First of all, the role of the team leader cannot be underestimated. In every team there should be a person who should act as a local team leader, sometimes taking the responsibilities of the project manager for the tasks like work assignment. Due to limited direct contact with the customer (visa restrictions) it was important that the person who was in charge of defining the scope of the project phase (requirements gathering and documentation) was able to work efficiently under time pressure. As it was described above, face-to-face contact was preferred way of communication, and the requirement gathering phase was found the hardest to perform as it was done remotely.

Another important factor was information sharing among team members and tools that were used for it. Team members were up to date with the current project activities as well as with the future plans for the project. It was done by setting up weekly 
teleconferences gathering all the people involved in the project and allowing to discuss current issues and actions. It was also important to grant access for everybody to necessary resources like corporation intranet, email, product documentation etc.. Lack of these facilities can lead to frustration and lower motivation of the team.

Finally, the most important success factor was unique set of people. It was not mentioned accidentally in the survey that one of the qualities that helped to overcome the distance was trust and commitment. When people can count and rely on each other they can perform very well despite the obstacles.

\section{References}

[1] Martin, R.C.: Agile Software Development, Principles, Patterns, and Practices. Prentice Hall, Englewood Cliffs (2002)

[2] Boehm, B., Turner, R.: Balancing agility and discipline. Addison-Wesley, Reading (2004)

[3] http://en.wikipedia.org/wiki/Wiki

[4] Basili, R.V., Shull, F., Lanubile, F.: Building Knowledge through Families of Experiments. IEEE Transactions on Software Engineering 25(4), 456-473 (1999)

[5] Orlikowski, W.J., Baroudi, J.J.: Studying Information Technology in Organizations: Research Approaches and Assumptions. Information Systems Research 2(1), 1-28 (1991)

\section{Appendix: Survey Structure}

This appendix reports the questions that composed the survey in the exact form as the survey respondents heard them during the interviews. The questions were divided into three categories.

\section{Method and Tools}

1. What methods and techniques (in terms of software development method) that you used in the project were particularly useful in a remote development setting?

2. Which tools you felt contributed to the information sharing (e.g. teleconferences, emails, chat, wiki, configuration management, etc.) and which did not? Please motivate your answer.

\section{Project Management Techniques}

3. What project management techniques (e.g. scope management, resource management, task and assignment management, delegation, customer relationship management) facilitated the interaction between the project manager and customer (located in the Middle East) and the team (located in Europe)? what instead did not work?

4. Was this project more agile or more disciplined? Why? How would you improve the approach?

5. Which phase of project management (scope management, time management, resource management, communication management, risk management, quality management, etc.) was made harder by the distance between you and the project manager and what instead was not influenced? 
6. What personal qualities of the project manager, architect and developers helped most overcome the distance? To what extent did the roles deviate from the job description? For instance, did the architect sometimes act as project manager?

\section{Cultural Differences}

7. What cultural differences did you notice (as far as work approach is concerned) in your multi-national team (please take into account the customer team as well)?

8. Which of the differences did you find stimulating and having good influence on project performance? Which not? Why?

9. Having the current experience in place would you change anything in project management approach, used tools or methodologies in order to diminish the negative / strengthen the positive influence of the cultural difference? 